\title{
ABSTRACTS OF THE MEETING OF THE CLINICAL GENETICS SOCIETY HELD ON 28 NOVEMBER 1990 AT THE INSTITUTE OF EDUCATION, LONDON
}

\author{
Analysis of SRY, the present \\ candidate testis determining factor \\ (TDF), in sex reversed patients and \\ evidence for novel regulation of \\ the zinc finger gene ZFY, the \\ previous candidate TDF \\ M NORTH, N AFFARA, \\ M A FERGUSON-SMITH \\ Department of Pathology, \\ Tennis Court Road, Cambridge \\ University, Cambridge CB2 IQP.
}

Three XX males and one XX intersex have previously been shown to contain only $35 \mathrm{~kb}$ of $\mathrm{Y}$ specific sequence (immediately adjacent to the pseudoautosomal boundary). A conserved open reading frame has been identified in this region and is proposed to encode a gene (SRY) which acts as a dominant male determinant by stimulating the embryonic 'indifferent' gonad to differentiate as testes. We have tested this hypothesis by examining a number of sex reversed patients for the presence of SRY and the Y pseudoautosomal boundary using the polymerase chain reaction. Significantly, we found six XX males who do not contain SRY or other $\mathrm{Y}$ sequences immediately proximal to the pseudoautosomal boundary. Since testis differentiation can occur without SRY, it is likely that further (possibly X linked) components of the testis determining cascade remain to be discovered. We have additionally shown that SRY (with one exception) is not deleted in a selection of XY females and we are at present searching for point mutations in these subjects which would result in SRY loss of function. Although the Y linked zinc finger gene $\mathrm{ZFY}$ is not present in the $35 \mathrm{~kb}$ interval discussed above, it may still be an important component of male development and has been strongly implicated in germ cell proliferation and differentiation. We report that, in adult testis, ZFY undergoes differential polyadenylation producing one transcript which contains possible fos-like $3^{\prime}$ instability determinants.
Identification and mapping of a potential zinc finger transcription activator gene on human

chromosome $\mathbf{X}$ which is expressed in adult testis

CAROLE SARGENT, JENNIFER

CHALMERS, DANIEL CROWTHER, SARAH LLOYD, JOHN GIBSON, SULTAN HABEEBU, NABEEL A AFFARA, MALCOLM FERGUSON-SMITH

Department of Pathology, University of Cambridge, Tennis Court Road, Cambridge.

We have screened an adult human testis cDNA library at low stringency with a genomic probe derived from the zinc finger domain of the $\mathrm{Y}$ linked gene ZFY to isolate further members of this class of gene. A total of 18 cross reacting cDNA sequences were isolated which appear to comprise a sub-family of zinc finger genes. Ten of these clones can be divided into four groups on the basis of insert size, and each group probably represents a separate cloning event of part of the same mRNA species. The two longest clones have been characterised by in situ hybridisation and nucleotide sequence analysis. CMPX1 (2.9 kb) maps to the $X$ chromosome at Xq13-q21.1 by in situ hybridisation. Southern blot analysis of a subject with an interstitial deletion of Xq21.1q21.3 further localises the gene to the interval. CMPX1 appears to be derived from an unspliced RNA species containing part of the zinc finger domain of the gene and an upstream splice junction in a position analogous to that in $\mathrm{ZFY} / \mathrm{ZFX}$. Both the putative intronic and exonic sequences map to the $X$ chromosome by somatic cell hybridisation and have been shown to be contiguous sequences by PCR amplification of genomic DNA. 5CMP1 (3.9 kb) encodes an acidic region and a nuclear localisation signal at a position analogous to that in ZFY, in addition to the zinc finger domain observed in CMPX1. Southern blot hybridisation of probes derived from the acidic domain to panels of somatic cell hybrid DNA and human male and female DNA confirms the localisation of these sequences to Xq21.1-q21.3. Overall, the zinc finger domain of CMPX1 is $75 \%$ homologous to ZFY/ZFX at both the nucleotide level and the amino acid level. It has the same characteristic organisation of the finger repeat units with either three or four amino acid residues between the his residues in alternating zinc fingers. In contrast, the acidic domain is only $4 \%$ homologous to ZFY/ZFX. This suggests that although the genes may have arisen from the same ancestral zinc finger domain by duplication, these have subsequently become associated with different acidic domains.

A physical map encompassing the Friedreich's ataxia locus DAVID WILKES*, JACQUI SHAW*, RAKESH ANAND $\dagger$, JOHN RILEY $\dagger$, PETER WINTER $\ddagger$, JULIE WALLIS*, ALBERT DRIESEL $\ddagger$, ROBERT WILLIAMSON*, SUSAN CHAMBERLAIN* ${ }^{*}$ Departments of Biochemistry and Molecular Genetics, St Mary's Hospital Medical School, Imperial College, London W2 1PG; +Biotechnology Department, ICI Pharmaceuticals, Mereside, Alderley Park, Macclesfield, Cheshire SK10 4TG; $¥$ Dechema Institut, Theodor-Heuss-Allee 25, 6000 Frankfurt am Main 97, West Germany.

Friedreich's ataxia is a recessively inherited neurodegenerative disorder affecting both the central and peripheral nervous system. The disease affects 1 in 50000 persons in the UK with a carrier frequency of 1 in 110 . Presenting usually before puberty, the disease is characterised by a progressive cerebellar ataxia, dysarthria, sensory loss, and diminished or absent tendon reflexes. We have previously mapped the Friedreich's ataxia locus (FRDA) to chromosome 9q13-21.1 using two anonymous markers D9S15 (MCT112) 
and D9S5 (DR47). Linkage analysis suggests that the locus lies within a 2 $\mathrm{Mb}$ interval including the marker loci, although absence of recombination in the region has prevented the precise positioning of $F R D A$. We report the construction of a physical map of the region, showing that the two marker loci lie within a $460 \mathrm{~kb}$ interval and identify CpG islands which may indicate the presence of coding sequence. Yeast artificial chromosomes (YAC) clones have been isolated spanning a $700 \mathrm{~kb}$ interval as part of the strategy to characterise the gene defect.

Mapping of the gene for multiple self healing squamous epithelioma to the long arm of chromosome 9

D R GOUDIE, M A R YUILLE, N A AFFARA, M A FERGUSON-SMITH Cambridge University, Department of Pathology, Tennis Court Road, Cambridge.

Multiple self healing squamous epitheliomas (MSSE) occur as an autosomal dominant trait, characterised by the development of skin tumours which are morphologically indistinguishable from sporadic squamous carcinomas. Despite their similarity to carcinomas of the skin (invading deep into the dermis and along local lymphatics) the lesions regress spontaneously, after a period of weeks or months, leaving disfiguring scars. Affected subjects may develop from one or two to over a hundred tumours in a lifetime. We report evidence of genetic linkage of the MSSE locus to D9S29 (LAMP92) and adjacent marker loci on the distal long arm of chromosome 9. We have studied 13 affected families (59 affected subjects and 160 of their close relatives) with 57 DNA, serological, and serum protein markers for polymorphic loci throughout the autosomes. No definite recombinants have been observed between the MSSE locus and D9S29. The maximum lod score at a recombination fraction of 0.02 is 8.05 . Studies with adjacent markers reveal that the MSSE locus is unlikely to lie distal to $O R M$ and D9S16.

\section{Linkage analysis of fragile}

$X$ syndrome using polymorphic distal Xq markers

I A GLASS*, R DEL MASTRO*, w G LANYON†, G MARSHALI $†$,
J A RAEBURN‡, M W KILPATRICK*, J M CONNOR†

${ }^{*}$ Department of Clinical Genetics, West Midlands Regional Genetics Service, †University Department of Medical Genetics, Glasgow; $\ddagger$ Department of Clinical Genetics, University of Nottingham.

Fragile $\mathrm{X}$ syndrome is the commonest form of inherited mental handicap and poses considerable difficulties in genetic counselling. To date, attempts at the application of linked RFLPs for genetic counselling of fragile $X$ syndrome have been hampered by the unusually high rates of recombination observed in distal $\mathrm{Xq}$ for the tested markers. Seventeen families segregating for fragile $\mathrm{X}$ syndrome were tested with the potentially closely linked polymorphic loci DXS369, DXS297, DXS296, and DXS304. Peak lod scores obtained by linkage analysis for markers detecting these loci were: RN-1 detecting DXS369, $\mathrm{Z} \max =3.02$ $(\theta=0.06)$; VK23B detecting DXS297, $\mathrm{Zmax}=2.92 \quad(\theta=0) ; \mathrm{VK} 21 \mathrm{~A} / \mathrm{VK} 21 \mathrm{C}$ detecting $D X S 296, Z_{\max }=3 \cdot 82(\theta=0)$; U6.2 detecting $D X S 304, Z \max =3.34$ $(\theta=0.04)$. Although no phase known recombination events were delineated in this study, apparent double recombination events with DNA markers closely flanking the mutation identified two non-penetrant males in one pedigree. This data set supports the notion that sufficient tightly linked flanking markers mapping around $F R A X A$ now exist for application (in combination with cytogenetic methods) for detection of both carrier females and non-penetrant males, and for prenatal diagnosis.

Mapping of the Von Hippel-Lindau (VHL) disease locus to a small region of chromosome 3p by genetic linkage

E R MAHER*, E BENTLEY*, J R W YATES*, F LATIF†, M LERMAN $\dagger$, B ZBAR $\dagger$, N A AFFARA*, M A FERGUSON-SMITH*

*Cambridge University Department of Pathology, Cambridge; +National

Cancer Institute, Frederick, USA.

Von Hippel-Lindau disease is a dominantly inherited cancer syndrome with variable expression. The most frequent manifestations are retinal and CNS haemangioblastomas, renal cell carcinoma, phaeochromocytoma, and renal, pancreatic and epididymal cysts. The VHL disease gene has been mapped to the short arm of chromosome 3 by linkage to the cRAF1 oncogene at 3p25 (Seizinger et al, Nature 1988;332:268-9; Maher et al, $\mathcal{F}$ Neurol Sci, in press). To localise the VHL disease gene further, we have performed genetic linkage studies in 22 families using polymorphic DNA markers from distal 3p. Significant linkage was detected between the VHL disease locus and $R A F 1$ ( $\mathrm{Zmax}=5.89$ at $\theta=0.06$, confidence interval $0.01-$ $0 \cdot 16)$, and $D 3 S 18(\mathrm{Zmax}=6.6$ at $\theta=0.0$, CI $0.00-0.06)$. The most likely order of markers is D3F15S2-THRB$R A F 1-D 3 S 18-(D 3 S 191, D 3 S 225)$ Hosoe et al, Genomics, in press). Our results localise the VHL disease gene within a small region (approximately 8 cM) of 3p25-p26 between RAF1 and (D3S191, D3S225), and close to the D3S18 locus.

\section{Evidence for a second genetic} locus for porphyrin metabolism on chromosome 11q

B NORTON*, W G LANYON†, M R MOORE‡, G R YOUNGs*, J E M TOMLINSON†, D A AITKEN†, J M CONNOR† *Chester Royal Infirmary, Chester; tUniversity Department of Medical Genetics, Duncan Guthrie Institute, Glasgow; $\neq$ Department of Medicine and Therapeutics, Western Infirmary, Glasgow.

McColl et al (Lancet 1985;ii:796-9) identified a previously unrecognised form of acute porphyria in a large family from Chester. Chester porphyria (concurrent porphyria, MIM 17601 ) is characterised by neurovisceral crises and biochemical changes combining the features of acute intermittent and variegate porphyrias. Linkage analysis was undertaken in this extensive five generation family with Chester porphyria using seven polymorphic markers. Markers from chromosomes 9 and 14 showed no evidence for linkage but, in contrast, a maximum lod score of 5.25 at 0.07 recombination (confidence interval 0.07 to 0.15 ) was observed with probe PICJ52.208M2 (DI1S351). This provides evidence for the assignment of the locus for Chester porphyria to chromosome 1lq and also provides a marker for the identification of presymptomatic at risk relatives. Porphobilinogen deaminase $(P B G D)$ also 
maps to 1 lq but at least six recombinants could be identified in this family using PBGD cDNA polymorphisms. Hence, a distinct locus, whose protein product is as yet unknown, is involved in Chester porphyria.

Confirmation of the gene location for facioscapulohumeral disease (FSHD) on chromosome 4

M UPADHYAYA*, W BROADHEAD*, M SARFARAZI $\dagger$, P LUNT $\ddagger$, M OWEN*, P HARPER*

*Institute of Medical Genetics, UWCM, Cardiff; $+U$ Conn, Department of Pediatrics, Farmington, CT, USA; $\ddagger$ Bristol Royal Hospital for Sick Children, Bristol.

Wijmenga et al recently reported linkage between FSHD and a chromosome 4 microsatellite marker Mfd 22 (D4S171) in nine informative Dutch families, obtaining a maximum lod score of $Z=6.34$ at $\theta=0.13$ (Lancet, Sept 1990). Using our linkage panel of 24 British families with FSH muscular dystrophy we have confirmed the Dutch findings and found two other probes, pH 30 (locus D4S139) and pEFD 139.1, to be more closely linked. In 140 scorable meioses from 20 informative families we found only two recombinants between $F S H D$ and D4S139. All families showed consistent evidence for linkage with lod scores for individual families ranging from $0 \cdot 20$ to $5 \cdot 9$. A pooled maximum lod score of $Z=38.4$ at $\theta=0.01$ was obtained. Previous evaluation of clinical parameters from our 11 largest families had suggested homogeneity, but also identified the two families which formed the opposite ends of a continuous clinical spectrum. Although the family with the youngest average age of onset included one of the recombinants, this family gave a maximum lod score of $Z=1 \cdot 04$ at $\theta=0 \cdot 10$, supporting the view that FSHD is a genetically homogeneous disorder. Similarly, families with subjects previously labelled as 'FSH type spinal muscular atrophy' show linkage results consistent with those labelled as 'muscular dystrophy'. The substantial lod score, lack of heterogeneity, and proximity of these probes to FSHD will allow their use in presymptomatic and prenatal testing.
Genetic linkage of Marfan syndrome to markers on chromosome 15 P TSIPOURAS*, M SARFARAZI*, A S DEVI*, B WEIFFENBACH†, M BOXER $\ddagger$

*Molecular Genetics Laboratory, Department of Pediatrics, University of Connecticut Health Center, Farmington, CT, USA; +Collaborative Research Inc, Waltham, MA, USA; ¥Section of Human Genetics, Ninewells Hospital and Medical School, Dundee, Scotland.

Marfan syndrome (MS) is a genetic disorder of the connective tissue with pleiotropic manifestations from the musculoskeletal, cardiovascular, and ocular systems. Various extracellular matrix components have been unsuccessfully suggested over the years as candidate molecules. We and others used genetic linkage studies as means of positional mapping of the disease gene and generated an exclusion map (Blanton et al. F Med Genet 1990;27: 73-7). Recently, Kainulainen et al ( $N$ Engl f Med 1990;323:935-9) reported genetic linkage of $M S$ to two chromosome 15 markers, D15S45 and D15S29, with a maximum $Z$ at $\theta=0.0$ for both -markers. We genotyped our panel of twelve MS families for a number of chromosome 15 probes, among them $D 15 S 45, D 15 S 29$, and D15S48. The total number of scorable meioses in our family panel was 110 . We obtained a maximum $Z=9.653$ at $\theta=0.03$ with probe CRI- L442 (D15S48). This probe identifies two MspI dimorphisms. Using D15S45 and D15S29, we obtained maximum $Z=0.744$ at $\theta=$ 0.23 and $Z=0.178$ at $\theta=0.29$, respectively. Our results are in contrast to the findings reported by Kainulainen $e t$ al regarding the distance of the MS gene from $D 15 S 45$ and $D 15 S 29$. Our results confirm that a MS gene is located in the long arm of chromosome 15.

The use of uncultured amniotic fluid in the early diagnosis of genetic disease

$M$ REBELLO, G HACKETT, J SMITH, F E LOEFFLER, S ROBSON,

N MACLACHLAN, R W BEARD,

R WILLIAMSON, C H RODECK,

D V COLEMAN, C WILLIAMS

St Mary's Hospital and Medical School, London W2; Queen's Charlotte's Maternity Hospital, London W6.

Prenatal diagnosis for genetic disease is normally carried out on DNA from chorionic villus samples (CVS), the main reason for this being the relative ease of obtaining adequate samples of DNA. CVS is, however, associated with an approximately $4 \%$ rate of pregnancy loss. It would therefore be an advantage to be able to carry out these diagnoses on DNA from uncultured amniotic fluid samples with the associated lower risk of pregnancy loss. We have explored the possibility of using uncultured amniotic fluid samples to provide DNA for prenatal diagnosis reliably and have shown that this can be achieved using the polymerase chain reaction and $Y$ specific oligonucleotides as well as oligonucleotides used for the diagnosis of cystic fibrosis. Twenty samples have been examined. A significant risk in the use of uncultured amniotic fluid samples is contamination of the sample with maternal DNA. We have used PCR with oligonucleotide primers for hypervariable repeat regions to exclude maternal and other contamination of the DNA from amniotic fluid samples. We have investigated 15 samples in this way and were unable to find contamination. In order to determine the sensitivity of the test for contamination we have purposely contaminated amniotic fluid with maternal blood and compared the results with cytogenetic studies on the same samples.

Genetic amniocentesis at 11 to 14 weeks: a clinical and cytogenetic evaluation

J H SMITH, M T REBELLO,

G A HACKETT, C T H GRAY,

D E ROONEY, F E LOEFFLER,

R W BEARD, D V COLEMAN

Departments of Obstetrics and

Cytogenetics, St Mary's Hospital, London W2.

Early amniocentesis was performed on 110 women at 11 to 14 weeks' gestation. Amniotic fluid was obtained in 105 patients. There were two miscarriages and no perinatal losses, a total postprocedural loss rate of $1 \cdot 8 \%$. In two pregnancies there was premature rupture of membranes, both pregnancies having normal outcomes. Karyotype analysis was accomplished in all 105 cases. Our results, and those in current world publications, indicate that early amniocentesis may have an increasing role to play in prenatal diagnosis. 
Does the trade off between the gestational age and miscarriage risk of a prenatal diagnostic test vary according to genetic risk?

R J LILFORD

St Fames's University Hospital, Leeds.

The genetic risk of patients choosing prenatal diagnostic tests varies widely. At one extreme, linkage studies for Huntington's chorea will fail to exclude the disease in half of the cases, while some women select invasive tests at a risk of Down's syndrome of 1 in 300 or even less. During a recent talk on prenatal diagnosis, I asserted that as genetic risk rises, so a higher procedure related abortion risk of an earlier gestational age test becomes more acceptable. However, a member of the audience questioned the logic of my argument and asked whether it followed that, all other things being equal, people with high genetic risk have more to gain from an earlier but riskier test than those of low genetic risk. Intuitively it seemed that the trade off in favour of an earlier test with a higher iatrogenic miscarriage risk increased with the likelihood that there would be a need to terminate the pregnancy on the basis of a positive result. However, the persistent questionner challenged the apparent implication that high genetic risk should increase acceptance of the risk of loss of a normal baby. The mathematical analysis presented here follows from this debate which challenged an assumption that had hitherto seemed self-evident in prenatal diagnostic circles.

IQ test results and educational achievements in unselected children with sex chromosome aneuploidy MIRANDA JONES, $S$ G RATCLIFFE MRC Human Genetics Unit, Western General Hospital, Crewe Road, Edinburgh.

To provide information for prenatal counselling, we present results from the neonatal screening of 34380 infants born in Scotland between 1967 and 1979 during which 58 subjects were found to have non-mosaic sex chromosome anomalies (SCA). Follow up data are available on 19 XYY and 19 $\mathrm{XXY}$ boys, and $16 \mathrm{XXX}$ girls, as well as on a control group of 149 children. Speech development was delayed in a higher proportion of SCA children than in controls, $44 \%$ of $X Y Y, 47 \%$ of $\mathrm{XXY}$, and $50 \%$ of XXX children receiving speech therapy compared with $11.2 \%$ of male and $5.4 \%$ of female controls. IQ was assessed in all children between 7 and 14 years using the Weschler Intelligence Scale for Children. The mean Full Scale score for XYY boys was $102 \cdot 3$, with a range from 74 to 121 ; XXY boys ranged from 67 to 131 with a mean of $94 \cdot 5$. The mean score for the XXX girls was $85 \cdot 2$, with a range from 64 to 110 . All group comparisons, after controlling for social class, showed significant reductions in both verbal and performance IQ for SCA children, while retaining a social class gradient. The incidence of remedial teaching was increased in all SCA groups, at $54 \%$ of $\mathrm{XYY}$ boys, $77 \%$ of XXY boys, and $81 \%$ of XXX girls, compared with $18 \%$ of male and $12 \%$ of female controls. On testing, reading deficits were found in SCA children at both 8 and 13 years. Number skills were lower at the age of 13 for XXY and XXX groups, but not in the XYY boys; 52 and 54 children attended normal schools. Of 26 SCA children who have left school, two $\mathrm{XXY}$ boys are at University and one is at Further Education College. An XYY boy is training to be a nurse, one XXX girl is at Art College, and another starts at FE college shortly.

Screening and genetic counselling for relatives of patients with breast cancer in a family cancer clinic

R S HOULSTON, S HARRINGTON, L LEMIONE, E MCCARTER, J SLACK Department of Clinical Genetics, Royal Free Hospital School of Medicine, London NW3 2QG.

From 253 consecutive pedigrees taken from patients with breast cancer relative risks were estimated for first degree relatives by life table methods. For relatives of index patients older than 55 the relative risk was 1.57 , if less than $552 \cdot 29$, and 3.84 for those relatives of index patients less than 45 . A family cancer clinic was set up for relatives of patients and breast screening offered based on estimated life time risk. Women with family histories compatible with the Lynch type II cancer family syndrome were offered additional screening for colon and pelvic tumours. Self referrals, as well as those patients referred by General Practitioners, were accepted into the programme. In three years 900 patients have been seen.

Non-penetrance in tuberous sclerosis DAVID W WEBB, JOHN P OSBORNE

Bath Unit for Research into Paediatrics, Royal United Hospital, Combe Park, Bath BA1 3NG.

The skipped generation is a well recognised feature of autosomal dominant conditions and should imply non-penetrance. It can only be considered to have occurred when the nonpenetrant subject has both an affected parent and an affected child. In addition, the non-penetrant subject must have been rigorously investigated to exclude reduced penetrance. Skipped generations have not been reported in tuberous sclerosis, but reduced penetrance is a well recognised feature of the disease. We report a family with a subject with reduced penetrance and another with non-penetrance between a great grandfather and his great grandson. The grandfather with reduced penetrance has probable cardiac rhabdomyomata and developed an ungual fibroma after the age of $\mathbf{4 0 .}$ His daughter is as close as we have seen to non-penetrance in this disease, with no evidence of tuberous sclerosis despite rigorous investigations including Wood's lamp, direct and indirect fundoscopy, cerebral CT scanning, magnetic resonance imaging, renal ultrasound, and echocardiography.

Testing children for late onset genetic disorders

P S HARPER, T COLE, A CLARKE Institute of Medical Genetics, University of Wales College of Medicine, Heath Park, Cardiff CF4 4XN.

The isolation of increasing numbers of disease genes and of closely linked DNA markers is making possible age independent genetic testing that has major implications for children in families with genetic disorders. We have already emphasised the ethical and other problems of childhood testing in Huntington's disease and urged the need for widespread debate in relation to other disorders. We present here a series of case reports arising from referrals during the current year, which illustrate the wide range of disorders involved, the need 
for careful individual consideration in each case of the factors for and against testing, and the frequency with which these issues are already arising in genetic counselling practice.

\section{Clinical genetics after the 1990 NHS Act \\ RODNEY HARRIS \\ Department of Medical Genetics, St Mary's Hospital, Manchester.}

Everyone is concerned about the effects of the reform of the NHS on genetic services for patients and families, on their personal working patterns, and on career prospects of trainees. I will summarise what is being done in the Royal College of Physicians of London and elsewhere to develop new opportunities and protect what has already been achieved. This is mainly about clinical genetics; the ACC and CMGS are looking after the laboratory aspects. District directors of public health and general managers are required by the new Act to assess the health needs of their resident populations and to purchase what they can afford. Geneticists must ensure that districts do not overlook the genetic needs of their populations and, possibly in competition with other potential providers, negotiate contracts to provide an agreed volume and quality of services. Working parties of the RCP and CGS will report by the end of 1990 on computers and software, on basic data sets to measure volume, cost, and quality, and on guidelines for health authorities on the genetic needs of their populations (reviewed in $\mathcal{F}$ Med Genet 1990;27: 711-4. The RCP has already sent guidelines on job descriptions to every consultant clinical geneticist to help them when they are negotiating their personal job plans and facilities with general managers. Consultant and junior clinical genetic posts are the responsibility of the RCP which has been very supportive, as the recent rapid increase in new consultant jobs shows. The Joint Planning Advisory Committee (JPAC) has also been sup-, portive and the number of $S R$ and registrar posts allocated to genetics has increased. This is partly because JPAC believes that paediatricians and others will require genetic training leading to the unfortunate phrase " $50 \%$ wastage of registrars in clinical genetics". This official terminology, the relative youth of the many new consultants, and the NHS changes may make juniors anxious about there being enough consultant posts in the future. The number of junior posts is in fact tied firmly to 'consultant opportunities' and a fall in new consultant jobs will, under JPAC rules, rapidly reduce the number of SR and registrar posts. As the new registrar posts are to be introduced over a period of several years and regions are not rushing to fund them, there should not be significant bottlenecks for trainees in clinical genetics.

\section{Posters}

The aetiology of hearing loss in a population of 204 institutionalised children

A M DEREYMAEKER, ${ }^{*}$ J P FRYNS*, J ARS†, A KLECZKOWSKA* ${ }^{*}$ Centre for Human Genetics, Catholic University of Leuven, Belgium, fInstitut Royale des Sourds et Aveugles (IRSA), Bruxelles, Belgium.

The aetiology of hearing loss in 204 (114 males, 90 females) inmates of the -IRSA (Royal Institute for Deaf and Blind Children) has been investigated with special reference to genetic factors. Finally, we selected 155 children for further investigation. Almost, one third of the inmates were children of immigrants (46 versus 109), the majority being of North African or Italian origin. The 109 Belgian children came from 103 families, of which two were consanguineous (1.9\%). The 46 immigrant children came from 42 families of which eight were consanguineous (19\%). The study indicated the following aetiological classification of the whole population investigated:

$\begin{array}{lc} & \text { Belgians (109) } \\ \text { Hereditary deafness } & 22 \cdot 9 \% \\ \text { Acquired deafness } & 44 \cdot 9 \% \\ \text { Unknown origin } & 32 \cdot 1 \% \\ & \\ & \text { Immigrants (46) } \\ \text { Hereditary deafness } & 39 \cdot 1 \% \\ \text { Acquired deafness } & 45 \cdot 6 \% \\ \text { Unknown origin } & 15 \cdot 2 \%\end{array}$

Autosomal recessive hearing loss was confirmed in 33 out of 155 pupils $(21 \cdot 3 \%) ; 17$ pupils were Belgian (15.6\% of 109$)$ and 16 were immigrants (34.8\% of 46). Usher syndrome was diagnosed in two patients and Alezzandrini syndrome in one other. Autosomal dominant hearing deficit was found in 10 of the 155 pupils $(6.4 \%)$; eight of these were Belgian (7.3\% of 109$)$ and two were immigrants $(4 \cdot 4 \%$ of 46$)$. Waardenburg syndrome was present in two children and Stickler syndrome in one other boy. We did not find any evidence for an $X$ linked type of deafness in the pupils in this study.

\section{Idiopathic arterial calcification: \\ a further six cases \\ T HUSSAIN*, w A PATRICK†, \\ A A GIBSONt, D R FITZPATRICK* \\ *Duncan Guthrie Institute, \\ Yorkhill, Glasgow G3 8SF; \\ †Department of Pathology, \\ RHSC, Yorkhill, Glasgow G3 8Sf.}

Idiopathic arterial calcification (IAC) is a disease of unknown aetiology characterised by widespread deposition of calcific material in the elastic membrane of arteries (excluding those in the brain). Early death is characteristic, caused by medial proliferation and luminal obstruction of the coronary arteries. The condition is listed in McKusick's recessive catalogue (MIM 20800) as there have been several reported sib recurrences. We report six cases of IAC that have been diagnosed in our Paediatric Pathology Department over the last 25 years. Four of these presented in the first two months of life with sudden 'collapse', respiratory distress and early death. The histological features mentioned above were most pronounced in the coronary arteries in these four cases. The two remaining cases had atypical features; one of them was stillborn with the calcification limited to the pulmonary vessels and extensive subperiosteal new bone formation, and the other presented at the age of 8 years with acute myocardial infarction caused by luminal narrowing associated with calcification and intimal fibrosis in the coronary vessels. Four of our cases appear to have the 'classical' findings of infantile IAC. The other two cases are sufficiently dissimilar to make 'lumping' inappropriate.

Autosomal recessive agnathiaholoprosencephaly: a discrete entity M E M PORTEOUS, J BURN Department of Human Genetics, University of Newcastle upon Tyne.

The association between holoprosencephaly and agnathia has been long 
recognised. All previously described cases have been sporadic apart from a sib pair reported by Pauli et al in 1983 who were subsequently shown to have an unbalanced 46,XX,t $(6 ; 18)(\mathrm{p} 24.1$; p11.21) translocation. Recently, we have been involved in counselling a non-consanguineous, white European couple who has lost two fetuses from consecutive pregnancies with agnathiaholoprosencephaly. Skin fibroblasts failed to grow in both cases but parental karyotypes were normal. We conclude that agnathia-holoprosencephaly may not always be sporadic and this should be taken into account when counselling families at risk.

\section{A Scottish child with Kabuki make} up syndrome

J C S DEAN*, S SIMPSON*,

G F COLE†, $K$ DUNNE†

Departments of Medical Genetics*

and Child Healtht, University Medical School, Foresterhill, Aberdeen.

Since its original description by Niikawa and Kuroki in 1981, the Kabuki make up syndrome has been reported in many Japanese children, but only 10 times in non-Japanese persons (once in a European). We report a six year old female with the typical facies, bilateral fifth finger clinodactyly, prominent fingertip pads, and a benign systolic murmur. At birth, a two vessel umbilical cord was noted and investigation showed a minor duplex collecting system in one kidney. Premature thelarche was recorded at the age of 14 months, but has regressed spontaneously. Her height, weight, and OFC are on the 50 th centiles and she is developmentally delayed. She has a right lateral gaze palsy, with Duane retraction phenomenon, and mild ataxia. The almost myopathic facial appearance with ophthalmoplegia and ataxia suggested a mitochondrial myopathy, but muscle biopsy at the age of 3 years 9 months was normal. It may be significant that whereas eight out of 11 non-Japanese patients have significant neurological dysfunction in addition to developmental delay, this is mentioned in only eight out of 62 Japanese patients. It may be that in non-Japanese patients, myopathic or neurological features are more prominent, perhaps because only the more severe cases are recognised at present.
MR with variable hydrocephalus and spastic diplegia:

a further family with Fried's

$\mathbf{X}$ linked retardation?

J S PATERSON, J L TOLMIE

Duncan Guthrie Institute of Medical Genetics, Yorkhill, Glasgow G3 8Sf.

A Scottish family with an $\mathrm{X}$ linked pattern of retardation is presented. Four affected males have been documented and two still living have been examined. At least one more is probable from the family history. They show a variable picture of hydrocephalus, spastic diplegia, epilepsy and mental retardation. None has had fragile $X$ chromosomes, testicular enlargement, or adducted thumbs. No affected females have been noted. The clinical picture in each individually would have been insufficient to make us suspect a genetic disease. There are many similarities between our family and another Scottish family described by Fried (1972). No common ancestor has been traced between the two families, back to 1860 .

A familial platelet disorder with tendency to develop leukaemia J S PATERSON*, J L TOLMIE*, A D J BIRCH†

${ }^{*}$ Duncan Guthrie Institute of Medical Genetics, Yorkhill, Glasgow G3 8Sf; tDepartment of Haematology, Falkirk and District Royal Infirmary, Falkirk.

Five subjects in a family with a dominantly transmitted platelet disorder were studied. Two of these developed haematological malignancies. In addition, a child is known to have died of leukaemia in a separate branch of the family but is not yet proven to have had the platelet disorder. Affected persons have severe bruising despite only mild thrombocytopenia; this is because of a functional defect involving platelet phospholipase activity in thromboxane production. Despite this, major clinical problems are not common. Two family members were initially diagnosed as having ITP with subsequent treatment with steroids and splenectomy, neither giving improvement. Both these women later developed leukaemia. One has chronic myelomonocytic leukaemia 20 years after splenectomy and the other had acute myelomonocytic leukaemia 11 years after splenectomy. She died three months after diagnosis. A delay in diagnosis may have occurred because of difficulty in distinguishing leukaemic signs of blood films from post splenectomy changes. It is not clear if splenectomy contributes to an increased risk of malignancy in this family but it is certainly a hazardous and unnecessary procedure which could have been avoided if the genetic nature of the condition had been appreciated. Similar families have been described by Downton et al (1985) (McKusick No 17342) and Najean et al (1990).

Von Hippel-Lindau (VHL) disease: a genetic study

E R MAHER*, L ISELIUS†, J $R$ W YATES*, M LITTLER†, C BENJAMIN‡, R HARRIS $\ddagger$, J SAMPSON§, A WILLIAMS $§$, M A FERGUSON-SMITH*, N MORTON† ${ }^{*}$ Cambridge University Department of Pathology; +CRC Research Group in Genetic Epidemiology, University of Southampton; $¥$ Department of Medical Genetics, University of Manchester; SInstitute of Medical Genetics for Wales, University of Wales College of Medicine.

Genetic aspects of VHL disease were studied in familial and isolated cases. Complex segregation analysis with pointers was performed in $\mathbf{3 8}$ kindreds with two or more affected members. Dominant inheritance with almost complete penetrance in the highest age classes $(0.96$ at 51 to 60 and 0.99 at 61 to 70 years) was confirmed and there was no evidence of heterogeneity between families ascertained through complete and incomplete selection. The point prevalence of heterozygotes in East Anglia was $1 \cdot 89 / 100000$ $(1 / 53000)$ persons with an estimated birth incidence of $2 \cdot 73 / 100000$ (1/36 000) live births. Reproductive fitness was $0 \cdot 83$. Direct and indirect estimates of the mutation rate were 4.4 (95\% CI 0.9 to 7.9$) \times 10^{-6} /$ gene/ generation and $2 \cdot 32 \times 10^{-6} /$ gene/generation respectively. No significant association was found between the origin of new mutations for VHL disease and parental age or birth order.

Audit of fragile $\mathbf{X}$ families with respect to carrier females

$S$ EDEES, J A RAEBURN

Interdisciplinary Centre for Medical

Genetics, City Hospital,

Hucknall Road, Nottingham NG5 IPB.

Effective identification of carriers in fragile $\mathrm{X}$ families, along with the offer 
of prenatal diagnosis, could reduce that type of mental handicap. We set out to ascertain the level of identification, counselling, and genetic follow up of at risk females (between 16 and 35 years), noting factors important in failure. A total of 23 families was studied, 53 positively identified females from 22 families were at risk, seven were phenotypically affected, and the remainder (46) had risks between $4 \%$ and $100 \%$ of being carriers. A further 32 females in four families seemed at risk but age details were unknown. Of the positively identified females (46), 28 had received counselling and 27 cytogenetic screening. The following factors led to failure of identification and counselling: (1) reluctance to involve the extended family; (2) counselling completed before the significance of transmitting males was recognised; (3) relatives out of the geographical area; (4) sections of the family failing to take up counselling; (5) failure to contact females of child bearing age.

Family resistance to further investigation was the largest block to counselling. Information leaflets and the greater use of genetic nurse specialists have made some impact in this group. Further measures which may improve the situation are (1) walk in community genetic clinics, (2) contacting the family doctor of possible carriers, alerting them to the risk, and offering genetic counselling. This could be carried out without breaches in confidentiality. Identification of at risk females in their early reproductive years would then facilitate prenatal diagnosis.

\section{Alteration of carrier risk in DMD by fetal dystrophin analysis \\ DAVID E BARTON*, LOUISE $\mathrm{V}$ B NICHOLSON†, ROBERT MCMAHON*, B C CLARE DAVISON*, MALCOLM A FERGUSON-SMITH* *East Anglian Regional Genetics Service, Molecular Genetics Diagnostic Laboratory, Department of Pathology, Cambridge University; + Muscular Dystrophy Group Research Laboratories, Regional Neurological Centre, \\ Newcastle General Hospital, Newcastle upon Tyne.}

This poster presents an interesting case study in which the proband's intermediate carrier risk was greatly reduced by a combination of molecular genetic studies and analysis of fetal dystrophin. The index case died of severe Duchenne muscular dystrophy (DMD) in 1965, aged 16 years. Although there was no family history of DMD, his sister (the proband) was given a risk of $22 \%$ because of a single raised creatine kinase (CK) reading taken from their mother at the age of 56. The proband's CK levels were slightly depressed (carrier:non-carrier ratio 1:1.6). After the birth of her first child (a healthy male) the proband chose to terminate three pregnancies when the fetal sex was determined to be male, had one spontaneous abortion at 10 weeks, one miscarriage after CVS, and gave birth to one healthy girl. An eighth pregnancy was referred to us in June of this year and RFLP studies showed that the fetus carried the opposite alleles at several DMD intragenic loci to that of the healthy boy. No deletion was observed using cDNA probes. A sample of muscle was taken from the terminated fetus and Western blot analysis for dystrophin was carried out. Monoclonal antibodies to the rod and C-terminus regions of dystrophin both indicated the presence of full length protein at an abundance of 20 to $60 \%$ of that found in normal adult skeletal muscle. This result is inconsistent with a diagnosis of DMD. This approach is generally applicable in DMD families and should be considered whenever a male fetus at intermediate risk is to be terminated.

\section{DMD carrier risk assessment using PFGE \\ ROBERT MCMAHON, \\ DAVID E BARTON, \\ MALCOLM A FERGUSON-SMITH \\ East Anglian Regional Genetics Service, Molecular Genetics Diagnostic \\ Laboratory, Department of Pathology, Cambridge University.}

Over the last two years this laboratory has provided an increasingly complete molecular genetic diagnostic service for the Xp21 linked muscular dystrophies. Successful counselling of families at risk from these conditions requires the accurate calculation of the probability that particular females carry the disease gene. To facilitate the effective targeting of restricted resources and to reduce unnecessary worry in patients, it is desirable to obtain risks of $>95 \%$ or $<5 \%$ routinely wherever possible.
This poster illustrates, by means of individual case studies drawn from the diagnostic work of the last 12 months, how pulsed field gradient electrophoresis (PFGE) can contribute to this aim. Particular attention is drawn to the use of PFGE in the establishment of unambiguous carrier risks for the mothers of isolated index cases. Traditionally this group presents a diagnostic problem since it is usually only possible to assign them intermediate risks, except in those rare cases when noninheritance or heterozygosity of RFLP bands associated with the disease can be proven. Since PFGE can detect altered size fragments caused by the deletion in heterozygous females directly, it allows for the unambiguous allocation of at risk females to carrier or non-carrier classes, and examples in which risk has been altered upwards as well as downwards are presented. Discussion is made of the part that PFGE can play in an integrated approach to carrier detection and some of the problems of interpretation and protocol are raised.

Genetic linkage of Charcot-MarieTooth disease (HMSN) in the South Wales population

JOHN C MACMILLAN, P S HARPER

Institute of Medical Genetics,

University of Wales College of Medicine, Heath Park, Cardiff CF4 4XW.

A population clinical, electrophysiological, and molecular study of the Charcot-Marie-Tooth phenotype in Mid and South Glamorgan identified 54 families in which at least one member had been diagnosed with this condition. In 23 families, affected subjects were concordant for the electrophysiological criteria for 'hereditary motor and sensory neuropathy type I' (HMSN I), while in four families affected subjects were discordant within the currently accepted classification.
Linkage analysis of $\mathbf{X}$ linked amelogenesis imperfecta MICHAEL J ALDRED*, PETER J M CRAWFORD†, ENRIQUETA ROBERTS*, CATHERINE M GILLESPIE*, NICHOLAS S T THOMAS*, IAIN FENTON*, LODEWIJK A SANDKUIJL*, PETER S HARPER*
*Institute of Medical Genetics, 
University of Wales College of Medicine, Heath Park, Cardiff; tDepartment of Child Dental Health, University of Bristol Dental School and Hospital, Lower Maudlin Street, Bristol.

Two families with $\mathrm{X}$ linked amelogenesis imperfecta (XAI) have beęn investigated using seven polymorphic DNA markers located in the distal region of the short arm of the $\mathrm{X}$ chromosome. Two point linkage analysis showed close linkage to $D X S 16$ with a maximum lod score of 6.34 at $\theta=0.03$, as well as to the markers DXS43 and DXS92. Multipoint marker ordering indicated the most probable order to be XpterDXS237 (GMGX9)-DXS143 (dic56)DXS85 (782)-DXS16 (XUT23)DXS43 (D2)-DXS207 (PA4B)DXS92 (XG16)-cen, corresponding to the known loci ordering based on physical mapping and other linkage analyses, with $X A I$ situated proximal to $D X S 85$ and distal to DXS16. XAI is most probably situated between the loci $D X S 85$ and $D X S 16$ identified by the probes 782 and XUT23 respectively and located at Xp22.3-Xp22.2. This localisation suggests that the gene locus controlling amelogenin synthesis is probably involved in the disease process.

A molecular deletion map of the $Y$ chromosome long arm

A J O'REILLY, N A AFFARA,

K KWOK, M MITCHELL,

R E MAGENIS, M A FERGUSON-SMITH

University of Cambridge Department of

Pathology, Tennis Court Road,

Cambridge CB2 1QP; University

Department of Medical Genetics,

Duncan Guthrie Institute of Medical

Genetics, Yorkhill, Glasgow G3 8SF.

The study of structural abnormalities of chromosomes plays an important role in the development of molecular and functional maps of the human genome. Cytogenetically, however, the differentiation of breakpoints is rather limited and only crude differences can be distinguished among a panel of patients deleted for various amounts of a given chromosome. The analysis of genomic DNA from a series of patients isodicentric for $\mathrm{Yp}$, using cloned $\mathrm{Yq}$ specific DNA sequences, has improved upon the cytogenetic resolution of $\mathrm{Yq}$ breakpoints. A congruent molecular deletion map (with the exception of two anomalies) has been assembled from this data, which will be of further use in (1) the characterisation of new Yq specific probes, (2) the characterisation of new patient material, (3) pulsed field mapping of this region of the genome, (4) the localisation of areas of Yq where breakpoints preferentially occur, (5) the correlation of structural and functional abnormalities of Yq. There is evidence for the presence of a factor involved in spermatogenesis on the proximal long arm of the $Y$ chromosome. Preliminary long range physical mapping studies, using pulsed field gel electrophoresis, have been carried out and there is evidence for the presence of two $\mathrm{CpG}$ rich islands in the euchromatic region of Yq. These studies have also shown that the distribution of probes on this panel is not random. Some areas are 'probe rich', while others are very poorly represented by cloned DNA.

Physical mapping of deletion

breakpoints in patients with $X$ linked ichthyosis

R S NEWMAN, N A AFFARA,

J $R$ W YATES, M MITCHELL,

M A FERGUSON-SMITH

University of Cambridge Department of Pathology.

Previous studies have shown that approximately $80 \%$ of patients with X linked ichthyosis have a total deletion of the steroid sulphatase (STS) locus which lies in Xp22.3-Xpter. We have shown here by Southern analysis that a common core of sequences are absent in $78.6 \%$ of our cases, suggesting that the deletion breakpoints may be highly clustered. To characterise the region in more detail a long range physical map of over $3 \mathrm{Mb}$ surrounding the STS locus was constructed using pulse field gel electrophoresis (PFGE). The map enabled the following order of sequences, tel-SI19-GMGXY3-[STS, GMGXY19]-GMGX9-[dic56,S112]cen. Probes flanking the STS gene, dic56, SI19, and CRI-232, were used to detect altered sized fragments in DNA, separated by PFGE, from 10 patients with STS deficiency. This information enables the positioning of deletion breakpoints and indicates a deletion size of $2 \mathrm{Mb}$ in most patients. The evidence supports the clustering of breakpoints. PFGE might have an application in the detection of carrier females who possess one normal and one deleted X chromosome. Five CpG islands have been positioned around the STS locus on the physical map. These may be associated with other loci in the region, such as mental retardation and Kallman syndrome.

\section{Molecular heterogeneity in MCAD} deficiency

A I F BLAKEMORE*, P C ENGEL $\dagger$, N GREGERSEN $\dagger$, $S$ KOLVRAAS, D CURTIS*

*The Sub-Department of Human Genetics and the Department of Molecular Biology and Biotechnology, University of Sheffield, Sheffield; ‡Laboratory of Molecular Genetics, Skejby Sygehus, Arhus, Denmark; SInstitute of Human Genetics, University of Arhus, Denmark.

Medium chain acyl-CoA dehydrogenase (MCAD) deficiency is an inherited disorder of fatty acid oxidation which has been implicated in up to $5 \%$ of SIDS. We report our recent advances in the study and diagnosis of MCAD deficiency. We have characterised 49 MCAD probands by the polymerase chain reaction for the common G985 mutation. Analysis of these probands and seven MCAD families by Southern blotting and PCR has shown a strong association between the G985 mutation and a particular haplotype. Nine of the 49 probands are compound heterozygotes for G985, with the other defective alleles unassociated with the particular haplotype; two further patients do not have the G985 mutation. We have estimated the frequency of the G985 mutation at 1 in 70 (six heterozygotes in 410 newborns) and calculate that the common G985 mutation accounts for some $85 \%$ of total mutations causing MCAD. Thus the likely frequency of the disease lies between 1 in 14500 (frequency of total MCAD alleles) and 1 in 18500 (frequency of G985 alleles). Prenatal, postmortem, and asymptomatic diagnoses of MCAD deficiency are now possible by molecular means. RFLP pedigree analysis is important both to exclude possible deletions and in families where another mutation is responsible for the disease. 
A 90kb DNA deletion associated with neurofibromatosis type 1 MEENA UPADHYAYA, ANNIA CHERRYSON, WENDY BROADHEAD, ALAN FRYER, DUNCAN J SHAW, SUSAN HUSON, P S HARPER

Institute of Medical Genetics, University of Wales College of Medicine, Cardiff.

Neurofibromatosis type 1 (NF1) is an autosomal dominant disorder with a prevalence of 1 in 3000 to 4000 of the population. We have screened a panel of 125 unrelated NF1 patients using DNA markers which map to the critical region of the gene, using both conventional and pulsed field gel electrophoresis, and have discovered one case in which mutation to the NF1 phenotype is accompanied by deletion of DNA. This deletion lies between probes $17 \mathrm{~L} 1 \mathrm{~A}$ and $\mathrm{AC5}$ in the critical region of chromosome 17 and is $90 \mathrm{~kb}$ in size. The subject showing the deletion is an isolated case and shows typical clinical features.

Physical mapping with DNA probes in the region 9q33-34 which show linkage to the tuberous sclerosis locus

R M HARRIS, N P CARTER,

$S$ DESAI, R M HAMPSON,

D R GOUDIE, N A AFFARA,

J R W YATES, M A FERGUSON-SMITH

Department of Pathology,

University of Cambridge.

Tuberous sclerosis (TSC) is an autosomal dominant disorder with a birth prevalence of at least 1 in 15000 . Tumour-like malformations (hamartomas) occur in the brain and other organs. The most serious manifestations are mental retardation, seizures, and intracranial tumours. The dermatological features include facial angiofibromas (adenoma sebaceum), hypopigmented macules, ungual fibromas, and shagreen patches. At least two independent loci appear to cause TSC, with evidence for one locus at $9 q 34$ near the Ableson oncogene and the other near D11S144 on 11q. An accurate map of the $9 q 33-34$ region is essential for the precise localisation of a gene for tuberous sclerosis, for which we decided to use a physical approach. We have started to construct a marker map using pulse field gel electrophoresis and preliminary results show that markers ORM and MCOA12 are within $250 \mathrm{~kb}$ of each other. These PFGE results have been complemented by the use of a panel of flow sorted dot blots of the derivative chromosomes from a series of cell lines having balanced translocations between $9 q 11.9 q 34.3$. Hybridisation of marker sequences to one or other of the chromosomes localises the marker proximal or distal to the breakpoint.

\section{A collaborative linkage study of tuberous sclerosis using a new approach to the heterogeneity problem \\ THE TUBEROUS SCLEROSIS \\ COLLABORATIVE GROUP}

Markers from distal $9 q$ and distal $11 q$ were typed in 88 multiplex families with tuberous sclerosis (TSC). The data were analysed using the 'imaginary chromosome' approach. Overlapping multipoint runs covering both regions were undertaken for each family and contiguous marker-marker intervals assembled into one array of lods. The arrays of lods for all families formed an input for analysis by HOMOG2. The results support locus heterogeneity ( $p<0.0001$ ) with locus 1 (TSCI) in the region of ASSG and MCT96.1 on 9q34 and a second locus, which was unlikely to lie in the region of chromosome 11 studied, that is between the loci D1 $1 S 84$ and DIIS147.

Two years' experience of a polyposis coli register

D MORTON, C MCKEOWN, J HAYDON, R CULLEN, J GIBSON, M KEIGHLEY, $M$ HULTEN, F MACDONALD

Regional Genetics Service and

Department of Ophthalmology, East Birmingham Hospital;

Department of Surgery, Queen Elizabeth Medical Centre, Birmingham.

The West Midlands Polyposis Coli Register was established in mid 1988 to improve the care of families with familial adenomatous polyposis; 57 families have been notified to the register and FAP confirmed in 51. Of 270 known family members, 169 had a prior risk of $50 \%$. Of those between 12 and 40 years, only $45 \%$ were undergoing appropriate bowel screening, in some cases because they were unaware of the risk, in others because they had been discharged prematurely, and in others because they had declined investigation. Compliance has improved since the register was established. Investigation for extracolonic manifestations of FAP has helped clarify risk assessment; 68 subjects have had indirect ophthalmoscopy for CHRPEs (congenital hypertrophy of retinal pigment epithelium) and 104 blood samples have been used for DNA analysis.

The Cardiff familial adenomatous polyposis register

I FENTON, A WILLIAMS, J MYRING, J R SAMPSON

Institute of Medical Genetics, University of Wales College of Medicine, Heath Park, Cardiff.

We have developed a register for familial adenomatous polyposis (FAP) which fulfils three main functions. (1) Facilitating extended family management. The organisational core of the register is a pedigree which can be displayed on screen, together with any stored clinical or DNA information, at any time. Data can be edited on the pedigree or in the text based database display. (2) Facilitating risk assessment. Linkage input files(incorporating age onset defined liability classes) can be generated quickly and easily from within the database, overcoming the usual error prone and tedious manual procedures. (3) Comprehensive administrative and clinical data storage. Recommendations from the Leeds Castle Group and members of the EuroFAP Concerted Action have been followed to produce a database storing information in a standardised format on symptomatology, surgical procedures, pathology, and extracolonic manifestations. This should prove valuable for centres undertaking clinical research or collaborating on a European basis.

The North-Western Regional Genetic Register: the first decade L KERZIN-STORRAR, A A KHAN, E A WATTERS, A COLLEY, G EVANS, T THAKKER, A NORMAN,

C BENJAMIN, T CLANCY,

A P READ, T ANDREWS, $M$ SUPER,

D CRAUFURD, D DONNAI,

H M KINGSTON, R HARRIS

Regional Genetic Register,

North-Western Regional Genetic Service, St Mary's Hospital, Manchester.

In 1980 the North-Western Regional Genetic Register was established with 
the aims of offering genetic counselling to extended families of probands referred to the department, and to offer annual follow up to these subjects. The ongoing contact through annual recall has enabled us successfully to offer advances in predictive, carrier, and prenatal testing to families counselled up to 10 years ago. At the Register's inception, Huntington's chorea, Duchenne and Becker muscular dystrophies, and adult polycystic kidney disease families were included. Neurofibromatosis I was added in 1988 followed by Von Hippel-Lindau disease, myotonic dystrophy, balanced chromosome translocations, Gorlin syndrome, neurofibromatosis II, and fragile $\mathrm{X}$ over the past year. Currently, 2300 subjects in 900 kindreds are recalled annually. Experience has shown that long term contact provides an effective means of offering testing and counselling at the appropriate ages and life events. Scientific advances have enabled us to clarify and, in many instances, to reassure relatives previously thought to be at risk. Family members are only included on the Register with their permission after they have been counselled. We feel that the supportive nature and the long term commitment of the Register service has contributed to its acceptance. In addition to providing a service, the Register has generated a number of genetic, clinical, and psychosocial research studies. The therapeutic, social, and preventative benefits of the Genetic Register have been made clear to us over the past decade; we hope that the considerable resources required will continue to be provided in this era of NHS change.

\section{The epidemiology of Huntington's disease \\ P S HARPER \\ Institute of Medical Genetics, UWCM, Cardiff.}

HD has been one of the most thoroughly studied mendelian disorders; around 50 prevalence estimates are available from differents parts of the world, including 16 from Britain. The possibility of defining specific marker haplotypes and the likely detection of specific mutations in the near future makes an accurate world wide picture of prevalence important. Collections of published data, together with unpublished surveys, suggest the following conclusions. (1) The prevalence in the UK is rather uniform, with most recent and complete surveys suggesting a prevalence of 5 to 10 per 100000 . (2) A comparable uniformity exists across Europe, with no clear northsouth or east-west gradient, in contrast to previous views. (3) $\mathrm{HD}$ is relatively frequent in several Asian populations and has been recorded in most other indigenous populations worldwide. This relative uniformity would support an origin from more than one original mutation; a single mutation in Europe would have to be extremely ancient to account for the observed distribution.

\section{A national protocol for predictive testing in Huntington's disease DAVID CRAUFURD, AUDREY TYLER On behalf of the UK Huntington's disease prediction consortium.}

The availability of linkage markers for Huntington's disease (HD) has made it possible to offer predictive tests to presymptomatic subjects at risk. Because of concern about the impact of such predictions on those involved, the procedure was confined at first to a few centres with a research interest in psychosocial aspects of HD. Demand for predictive testing has been smaller than was initially anticipated and there are still relatively few data about the long term consequences for those given unfavourable results, even though it is now more than three years since the first tests in this country were carried out. However, the understandable reluctance of those who want tests to wait any longer has led to the introduction of predictive testing for HD in many genetic centres around the country. By the end of 1988 more than 150 predictive tests had been completed in the UK. In January 1990 a seminar was held in Manchester to see whether a consensus could be found about the most appropriate methods for preparing and supporting those who choose to have predictive tests. The meeting was attended by 19 representatives from 13 centres. It was agreed that participating centres (and any others who wished to join) would adopt a common 'core' protocol in order to ensure uniform standards of clinical care and to facilitate collaborative research in the future. Details of the agreed protocol are available from the authors.
Attitude of young people to carrier screening for cystic fibrosis E COBB, S HOLLOWAY, R ELTON, J A RAEBURN

Department of Human Genetics, Western General Hospital, Crewe Road, Edinburgh.

Following upon identification of the cystic fibrosis (CF) gene, there has been much debate about screening the general population for heterozygosity. At present, there is no consensus about when and where such screening should occur, but since one option is offering the test to senior school students, we assessed whether a sample of Edinburgh secondary school children were capable of understanding basic facts about CF and formulating opinions on carrier screening and prenatal diagnosis. Initially the students were given a short talk by one of us (EC) covering the nature of $\mathrm{CF}$, genetic principles, and prenatal diagnostic methods. Following this, $71 \%$ of pupils questioned clearly understood the main aspects of each of these topics. Examining the attitudes of these pupils to carrier screening and prenatal diagnosis, $86 \%$ were in favour of the principle of routine carrier screening and $88 \%$ thought that prenatal diagnosis should be offered to carrier parents. This study thus shows that third year secondary school pupils are capable of understanding the principles underlying tests of heterozygosity and, given that understanding, the majority are in favour of routine carrier screening for CF.

\section{Simple detection of the $\Delta F 508$} deletion in cystic fibrosis using PCR analysis on agarose

D S PLAHA, G H LINFORTH,

D P DUCKETT, I D YOUNG,

R SCOTT-JUPP, M S TANNER, A J JEFFREYS

Molecular Genetics Laboratory, The Leicester Royal Infirmary, Leicester.

A simple PCR technique is described in which amplification with primers flanking the $\Delta F 508$ mutation is followed by separation and direct visualisation of the alleles on $6 \%$ FMC Nusieve agarose, a safer alternative to the commonly used acrylamide. The $98 \mathrm{bp}$ normal and the 95 bp $\triangle F 508$ PCR products are very clearly resolved and, in heterozygotes, a heteroduplex is 
seen as an extra band above the 98 bp product. By repeatedly using the gel, a major advantage with agarose, approximately $100 \mathrm{CF}$ patients were successfully analysed on just two gels. This makes the technique very economical and appropriate both to a diagnostic laboratory and a screening programme.

Molecular diagnosis of cystic fibrosis STEWART J PAYNE*, DAVID E BARTON*, ANTHONY HEELEY†, JOHN R W YATES*, MALCOLM A FERGUSON-SMITH*

${ }^{*}$ East Anglian Regional Genetics Service, Molecular Genetics Diagnostic Laboratory, University of Cambridge, Department of Pathology, Tennis Court Road, Cambridge CB2 1QP; +Department of Clinical Chemistry, Peterborough District Hospital, Thorpe Road, Peterborough.

This laboratory provides a molecular diagnostic service for cystic fibrosis (CF). We have analysed $321 \mathrm{CF}$ chromosomes and found the common CF mutation ( $\triangle \mathrm{F} 508)$ on $253(78 \cdot 8 \%)$. We have provided ' 24 hour' prenatal diagnosis for CF using PCR with individually dissected chorionic villi as a DNA source. This approach is very rapid and avoids maternal cell contamination. A retrospective study of all CF children born in the region during the last 10 years has been carried out using Guthrie cards as a source of DNA for PCR. These cards have been collected in the regional immunoreactive trypsin screening programme. Frequency of $\Delta \mathrm{F} 508$ is similar to the general CF population $(79 \cdot 4 \%)$. There is no correlation between disease severity and possession of the $\Delta F 508$ mutation in this group.

Leukaemia mortality among relatives of cystic fibrosis patients in Wales: update 1990

L N AL-JADER*, $\mathbf{R}$ R WEST $\dagger$, J A HOLMES‡, L MEREDITH*, M C GOODCHILD ,, P S HARPER* University of Wales College of Medicine, Cardiff, *Institute of Medical Genetics, †Department of Epidemiology, $¥$ Department of Haematology, \Department of Child Health.

A total of 219 families of patients with cystic fibrosis living in Wales has been studied for the occurrence of other diseases and for cause of death. Our findings in relation to leukaemia show that there were eight deaths from leukaemia, five of the myeloid type, in the first and second degree relatives, significantly more than the expected on the basis of national age specific mortality rates. In comparison, mortality from all causes among sibs, parents, aunts, uncles, and grandparents was within the expected range. Screening the five myeloid leukaemic patients for the $\Delta$ F508 mutation showed that four were carriers of this mutation. We conclude that carriers of the $\Delta$ F508 mutation may have an increased risk of developing acute myeloid leukaemia. This could happen through the direct effect of the CF gene itself, or through its influence on another gene, such as the MET oncogene, or gene(s) involved in granulocyte function on the long arm of chromosome 7 .

\section{Register of developmental dental} anomalies: a computer program for recording clinical and DNA data and displaying pedigrees relating to inherited dental disorders M J ALDRED*, P J M CRAWFORD $\dagger$, I FENTON*, A CLARKE*

*Institute of Medical Genetics, University of Wales College of Medicine, Heath Park, Cardiff; tDepartment of Child Dental Health, University of Bristol Dental School and Hospital, Bristol.

We have established a Register of Developmental Dental Anomalies to record details of persons and families with inherited disorders affecting the teeth (their number, form, and structure) and oral and circumoral tissues together with other developmental conditions with no obvious genetic basis. The ectodermal dysplasias, and particularly X linked hypohidrotic ectodermal dysplasia, form a significant part of the Register. Amelogenesis imperfecta (AI) is a group of conditions affecting tooth enamel with autosomal dominant, autosomal recessive, or $\mathrm{X}$ linked modes of inheritance. Qualitative and/or quantitative defects result in the various phenotypes recognised. The prevalence of all forms has been estimated to lie between 1:700 and 1:14 000. Dentinogenesis imperfecta (DI) may be a feature of osteo- genesis imperfecta or may occur as an isolated trait with an estimated prevalence of 1:12 000. In both forms the structure of the dentine is abnormal. A pool of three and four generation families with either AI or DI suitable for linkage analysis has been collected together with a number of smaller pedigrees. To this end we have developed a computer program capable of allowing efficient access to and retrieval of records. Data recorded include personal details, pedigree information for subsequent graphical display and editing, any amount of DNA marker data, together with diagnosis and other relevant clinical information. This program allows lists to be generated using multiple searching criteria incorporating extensive use of Boolean logic where necessary. The editing of the data can be performed either conventionally on a text based screen or, more efficiently, on the actual display of a pedigree. This new feature reduces the potential error inherent in entering data on text only screens. The program has many tools to aid the correct specification of the codes necessary for pedigree generation and once displayed on screen any data field can be shown underneath the pedigree symbols and edited without reverting to text mode.

A new perspective on data storage problems associated with using PCR technology for linkage analysis M SARGEANT*, I FENTON† *Department of Psychological Medicine, University of Wales College of Medicine, Cardiff; IInstitute of Medical Genetics, University of Wales College of Medicine, Cardiff.

The number of loci described in human genetics is increasing rapidly, and with greater use of PCR technology will only continue to grow. The number of alleles specified at each locus is very likely to escalate. Current computer storage methods used in linkage analysis front end databases, while being adequate for standard Southern blotting/RFLP techniques, are not prepared for the data handling problems that will occur as PCR technology becomes the predominant laboratory technique. Storage of allele systems at present are based on the technique of coding lengths into simple numerical order. Hence the shortest fragment would be coded as a 1 , the 
next longest as a 2, and so on. The problems this will cause will be greatly exacerbated as PCR technology is used more frequently. To circumvent this we store the dinucleotide repeat alleles directly as alleles into a computer program, thus enabling easy comparison of diverse data sets from different centres. The widespread acceptance of PCR techniques will only aggravate the problem. To show the need for such systems we have reworked the classic HLA typings sib-pair analysis paper as described by Cudworth and Woodrow (BMF, 1975), and later reanalysed by Green and Woodrow (Tissue Antigens, 1977). The 1977 reanalysis was done manually, whereas our analyses have been performed totally in software using direct data entry. Our work so far has shown the depth of the analysis techniques and the large amount of time that can be saved by better automation of the methods; there are further gains to be had from the improving programming technologies as they are developed. The ability to automate much of the data storage not only saves considerable time, but more importantly allows the automatic checking of data for accuracy which would be highly error prone for the human eye. The value of such an automated system is confirmed by finding apparent inconsistencies in the coding schemes of the original papers mentioned above. We hope to extend this work to other linkage methods. The advantages for the busy laboratory worker should be obvious in that the original data themselves are worked with rather than a re-coded form of them. If successful, this technique may significantly reduce errors in these forms of linkage studies.

\section{REFER version 2: a computer program for formatting and manipulating references from the MEDLINE and REFERENCE UPDATE systems \\ I FENTON*, J ALDRED*, P J M CRAWFORD†, S BRIGANTI†, M SARFARAZI $\ddagger$}

*Institute of Medical Genetics, University of Wales College of Medicine, Heath Park, Cardiff; tDepartment of Child Dental Health, University of Bristol Dental School and Hospital, Bristol; $¥ D i v i s i o n$ of Human Genetics, Department of Pediatrics,

University of Connecticut, Farmington, USA.

The Vancouver style of references for medical and dental papers has been adopted by a number of journals, but it is still necesary to reformat references to accommodate the differing requirements of many publications. For some time we have successfully used a computer program (REFER version 1) capable of searching for manually entered references according to various criteria and automatically manipulating these to suit a particular journal. The program is capable of formatting references in all the major journal styles and new ones can be incorporated in the program by the user at any time. Hence, if selected papers from a bibliography are to be appended to a manuscript, all the references can be easily reprinted in the correct format for a particular journal without any retyping being necessary. This package has now been upgraded to permit direct entry of references downloaded onto floppy disk from a CD-ROM based search on MEDLINE, or from the floppy disk based version of REFERENCE UPDATE. It is thus possible for all references on MEDLINE (from 1966 onwards) covering a given topic to be entered into the program without needing to type them in. In addition, the facility to access REFERENCE UPDATE enables active researchers to add all recent publications in their particular field of interest to their references database between one and three months before they would be available on MEDLINE. This last feature is particularly relevant given the rapid advances in scientific knowledge in general, and in the field of genetics in particular. Until such time as a single referencing style is adopted by all editors we believe this software offers significant savings in time for authors in the preparation of manuscripts.

\author{
A database for dental genetic \\ disorders capable of displaying dental \\ images \\ I FENTON*, M J ALDRED*, \\ P J M CRAWFORD $\dagger$ \\ *Institute of Medical Genetics, \\ University of Wales College of Medicine, \\ Cardiff; + Department of Child Dental \\ Health, University of Bristol Dental \\ School, Bristol.
}

We have developed a computer program for recording dental hard tissue pathology. This can be applied in a fixed situation using a desktop computer, or in a variety of clinical locations using a portable computer. It has been developed to record personal details as well as clinical and radiographic findings. Also included is a checklist of further investigations that may be undertaken. The program makes it possible to generate dental images representing teeth present or absent together with variable shading to indicate severity of involvement of individual teeth, groups of teeth or the entire dentition. Additional fields of data entry allow for information from research publications to be compiled into the database to build up a comprehensive data resource relating to any particular condition. This last facility offers an efficient means of compiling a database which can then be searched on single or multiple criteria to reveal quickly and simply the features associated with clinical disorders. This can be used to aid in the differential diagnosis of particular clinical problems in dental disorders. The programming language used is Clipper, which, being a compiled superset of the dBASE language, means that the program is fast and allows the data to be interchanged with any other commercial software, such as statistical packages using dBASE files. 